\title{
Composição e diversidade da ictiofauna em riachos do Cerrado, bacia do ribeirão Ouvidor, alto rio Paraná, Goiás, Brasil
}

\author{
Nicelly B. Araújo ${ }^{1} \&$ Francisco L. Tejerina-Garro ${ }^{2}$ \\ ${ }^{1}$ Programa de Pós-Graduação em Ecologia e Produção Sustentável, Universidade Católica de Goiás. Campus II, Avenida \\ Engler, 74605-010 Goiânia, Goiás, Brasil. E-mail: nicellybraudes@hotmail.com \\ ${ }^{2}$ Centro de Biologia Aquática, Universidade Católica de Goiás. Campus II, Avenida Engler, 74605-010 Goiânia, Goiás, Brasil. \\ E-mail: garro@ucg.br
}

\begin{abstract}
Fish diversity and composition in Cerrado streams, Ribeirão Ouvidor basin, upper Paraná River basin, Goiás, Brazil. This study aims to describe the fish assemblage using ecological descriptors (richness, abundance, diversity of Shannon-Wiener, uniformity, relation richness/abundance and constancy. Fish sampling were carried out every two month from August 2004 to June 2005 in a stretch of 50 m long in nine tributaries and in the main channel of the Ribeirão Ouvidor. Four thousand forty-nine fish were collected using electric fishing equipment. These individuals are distributed in 35 species, six Orders and fourteen families. The most abundant species were Astyanax cf. fasciatus Cuvier, 1819 (762 individuals), Poecilia reticulata Peters, 1859 (725) and Astyanax altiparanae Garutti \& Britski, 2000 (650). The correlation between the channel width and the richness suggests that width streams has greatest richness that those with narrow channels. Only the diversity of Shannon-Wiener showed significant differences among streams. Astyanax cf. fasciatus and Rhamdia quelen Quoy \& Gaimard, 1824 were present in all the streams sampled, whereas the greatest number of constant species was found in the Santo Antonio stream.
\end{abstract}

KEY WORDS. Central Brazil; ecological descriptors; neotropical fish; Paranaíba river system.

RESUMO. Este estudo tem como objetivo descrever ecologicamente a ictiofauna quanto à riqueza, abundância, diversidade de Shannon-Wiener, uniformidade, relação riqueza/abundância e constância. As amostragens da ictiofauna foram realizadas bimestralmente de agosto de 2004 a junho de 2005 num trecho de 50 m de comprimento em nove afluentes e na calha principal do ribeirão Ouvidor, bacia do Alto Paraná. A coleta dos peixes foi realizada utilizando-se a pesca elétrica. Foram coletados 4049 indivíduos distribuídos em 35 espécies, seis ordens e 14 famílias. As espécies mais abundantes na bacia foram Astyanax cf. fasciatus Cuvier, 1819 (762 indivíduos), Poecilia reticulata Peters, 1859 (725) e Astyanax altiparanae Garutti \& Britski, 2000 (650). A correlação encontrada entre a largura da calha e a riqueza sugere que riachos largos apresentam maior riqueza que estes estreitos. Apenas a diversidade de Shannon-Wiener variou significativamente entre os riachos amostrados. Astyanax cf. fasciatus e Rhamdia quelen Quoy \& Gaimard, 1824 estiveram presentes em todos os trechos considerados, enquanto que a maior quantidade de espécies constantes foi encontrada no riacho Santo Antônio.

PALAVRAS-CHAVE. Descritores ecológicos; peixes neotropicais; região Centro-oeste; sistema do rio Paranaíba.

Apesar da extensa rede hidrográfica representada pelos riachos nas bacias hídricas brasileiras, poucos são os estudos realizados envolvendo a ictiofauna dos mesmos. Na bacia do rio Paraná, da qual faz parte o ribeirão Ouvidor, os estudos se concentram na sua região alta localizada no estado de São Paulo (CAstro \& Casatti 1997) num afluente do rio Pardo, 19 espécies; Luiz et al. (1998) nos riachos Água Sumida e Arigó, 41; Lemes \& Garutti (2002) num curso de água de cabeceira, 21; Braga (2004) na microbacia do ribeirão Grande, 37 ou no estado do Paraná (Penczak et al. 1994) nos tributários Caracu e Água do Rancho, 28 espécies; Shibatta \& Cheida (2003) em afluentes do rio Tibagi, 43.

Na bacia do rio Paraná, em Goiás, apenas dois estudos sistematizados abordando a ictiofauna de riachos foram desenvolvidos. O primeiro realizado por FialHo et al. (2007), sobre a estrutura das assembléias de peixes em 31 tributários da bacia do rio Meia Ponte, onde encontraram uma riqueza igual a 59, e o segundo por BENEDITo-CECílio et al. (2004) que realizaram um estudo da composição e estrutura em três cursos de água no Parque Nacional das Emas e encontraram sete espécies. Até o 
momento, nenhum estudo sistematizado da ictiofauna foi desenvolvido na bacia do ribeirão Ouvidor, a qual apresenta a mineração como principal atividade econômica (GALINKIN 2003). No entanto nesta bacia, como ocorre em todo o estado de Goiás, também são desenvolvidas atividades agropecuárias (NovAEsPINTO 1993). Estas atividades implicam no uso dos recursos hídricos, inclusive para consumo humano, o que levou à elaboração em 2004 de um projeto de estudo ambiental de maneira a subsidiar um Plano de Gestão Ambiental para a bacia.

Desta maneira, este trabalho objetiva responder às seguintes questões: I) Quais as espécies de peixes encontradas na bacia do ribeirão Ouvidor? II) A ictiofauna dos cursos d'água amostrados apresentam semelhanças quanto à riqueza, abundância, diversidade de Shannon-Wiener, uniformidade e relação riqueza/abundância? III) Quais as espécies constantes na bacia do ribeirão Ouvidor e nos cursos de água amostrados?

\section{MATERIAL E MÉTODOS}

\section{Área de estudo}

A bacia do ribeirão Ouvidor (Fig. 1) está localizada a leste da cidade de Catalão, no sudeste do estado de Goiás e abrange os municípios de Ouvidor, Três Ranchos e Cumari. O ribeirão Ouvidor nasce da junção dos riachos Taquara I e Taquara II e deságua no rio Paranaíba, fazendo assim parte da bacia do Paraná, o segundo maior sistema de drenagem da América do Sul (LowEMCConnell 1999). Os tributários do ribeirão Ouvidor apresentam a calha principal estreita (média $=3,99 \mathrm{~m} ; \mathrm{DP}= \pm 2,2$ ) e pouco profunda (média $=39,10 \mathrm{~cm}$; DP $= \pm 16,5$ ) (Tab. I). O substrato da calha é formado predominante por areia associada a cascalho (riachos Buraco, Olhos d'água, Posse dos Rodrigues e Riacho), a afloramentos rochosos (Lagoa, Ouvidor e Taquara II) ou a lama (Taquara I). A bacia não apresenta uma planície de inundação, sendo que a região drenada é coberta por vegetação do tipo Cerrado, que se caracteriza por apresentar diferentes fitofisionomias, entre as quais a vegetação ripária, a qual está estreitamente relacionada com o meio aquático (RiberRo 1998), e é intercalada na bacia em estudo, com áreas de pastagens (ribeirão Ouvidor, riachos Posse dos Rodrigues, Riacho, Olhos d'água), e industriais (riachos Taquara II e Santo Antônio). Porém, entre os cursos de água amostrados há estes que estão fragmentados pela presença de reservatórios (riachos Taquara I, Buraco, Sapê), recebem esgotos domésticos (riacho Santo Antônio) ou sofrem a influência de áreas urbanas (riacho Lagoa).

\section{Protocolo amostral}

As amostragens foram desenvolvidas em nove afluentes e na calha principal do ribeirão Ouvidor. As mesmas foram realizadas bimestralmente de agosto de 2004 a junho de 2005 durante um período sazonal (estiagem e chuva). Inicialmente, em cada riacho demarcou-se um trecho de $50 \mathrm{~m}$ de comprimento com auxílio de um marcador de distâncias (Topofil). Este trecho foi georeferenciado (GPS 12 Garmin) (Tab. I) e balizado utilizandose fitas plásticas de maneira a ser identificado ao longo das amostragens.

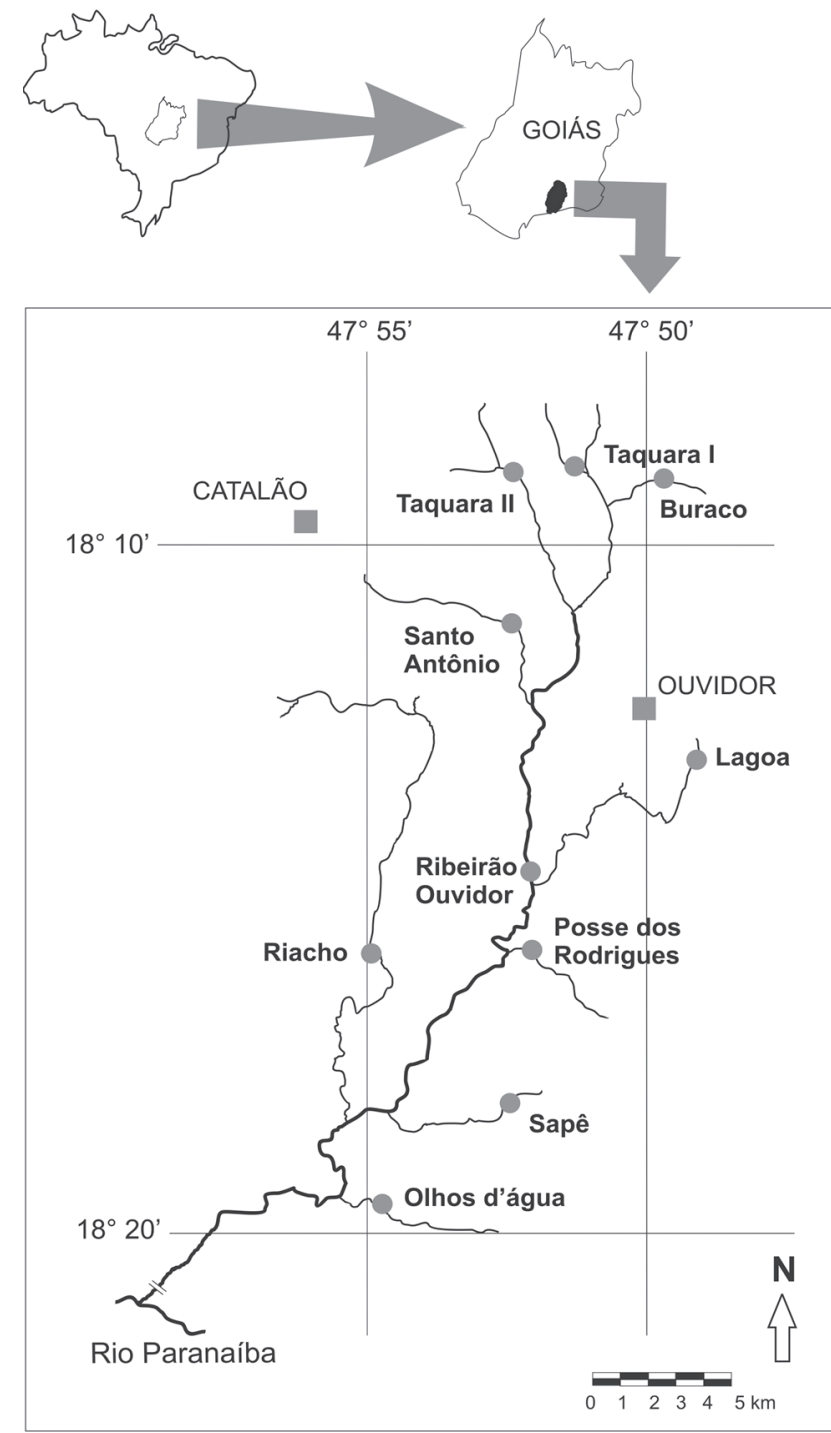

Figura 1. Localização dos trechos amostrais (círculos) nos cursos de água da bacia do ribeirão Ouvidor, alto rio Paraná, Goiás. Os quadrados indicam as principais cidades.

O método utilizado para a amostragem dos peixes foi o da pesca elétrica modificado de MAzzoni et al. (2000). Este é eficiente na captura de peixes de pequeno porte (SEVERI et al. 1995) como é o caso dos indivíduos encontrados na área de estudo, mas pode ter sido influenciado pela condutividade, a qual variou entre os trechos amostrados. Três pessoas percorreram o trecho de $50 \mathrm{~m}$ por três vezes conforme sugestão de EsTEvEs \& LOBÓN-CERviá (2001), no sentido jusante-montante. Desta maneira, o esforço amostral foi de três pessoas por duas horas e três passadas por $50 \mathrm{~m}$,

Os peixes capturados foram fixados em formol a $10 \%$ e colocados em sacos plásticos identificados, os quais foram posteriormente acomodados num tambor plástico contendo formol 
Tabela I. Localização e média dos dados de velocidade, largura e profundidade dos trechos nos riachos amostrados na bacia do ribeirão Ouvidor, alto rio Paraná, Goiás.

\begin{tabular}{|c|c|c|c|c|c|}
\hline Cursos de água & & ização & Velocidade $(\mathrm{cm} / \mathrm{s})$ & Largura (m) & Profundidade $(\mathrm{cm})$ \\
\hline Buraco & $18^{\circ} 09^{\prime} 40,2^{\prime \prime}$ & $47^{\circ} 49^{\prime} 46,1^{\prime \prime}$ & 18,15 & 1,13 & 13,00 \\
\hline Lagoa & $18^{\circ} 15^{\prime} 16,7^{\prime \prime}$ & $47^{\circ} 49^{\prime} 25,6^{\prime \prime}$ & 41,93 & 3,94 & 40,83 \\
\hline Olhos d’água & $18^{\circ} 23^{\prime} 21,3^{\prime \prime}$ & $47^{\circ} 54^{\prime} 28,8^{\prime \prime}$ & 46,03 & 2,42 & 13,56 \\
\hline Ouvidor & $18^{\circ} 17^{\prime} 47,6^{\prime \prime}$ & $47^{\circ} 52^{\prime} 48,7^{\prime \prime}$ & 54,86 & 8,89 & 51,49 \\
\hline Posse dos Rodrigues & $18^{\circ} 18^{\prime} 48,2^{\prime \prime}$ & $47^{\circ} 55^{\prime} 15,8^{\prime \prime}$ & 30,43 & 3,59 & 21,69 \\
\hline Riacho & $18^{\circ} 18^{\prime} 09,0^{\prime \prime}$ & $47^{\circ} 52^{\prime} 49,8^{\prime \prime}$ & 68,98 & 5,80 & 44,69 \\
\hline Sapê & $18^{\circ} 21^{\prime} 28,2^{\prime \prime}$ & $47^{\circ} 53^{\prime} 30,0^{\prime \prime}$ & 8,97 & 4,10 & 47,64 \\
\hline Santo Antônio & $18^{\circ} 12^{\prime} 18,6^{\prime \prime}$ & $47^{\circ} 52^{\prime} 34,9^{\prime \prime}$ & 20,07 & 4,59 & 50,56 \\
\hline Taquara I & $18^{\circ} 09^{\prime} 32,3^{\prime \prime}$ & $47^{\circ} 51^{\prime} 08,5^{\prime \prime}$ & 29,38 & 2,17 & 54,42 \\
\hline Taquara II & $18^{\circ} 09^{\prime} 16,6^{\prime \prime}$ & $47^{\circ} 52^{\prime} 37,1^{\prime \prime}$ & 20,42 & 3,27 & 53,14 \\
\hline
\end{tabular}

a $10 \%$ e transportados ao laboratório do Centro de Biologia Aquática da Universidade Católica de Goiás. Os peixes capturados foram triados, identificados com auxílio de chaves taxonômicas, sendo a confirmação da identificação feita mediante envio de exemplares para o laboratório de Ictiologia do Museu de Ciências e Tecnologia da Pontifícia Universidade Católica do Rio Grande do Sul.

Em cada trecho e a cada coleta foram medidas a largura da calha (m), a profundidade da água (m), ambas utilizando-se uma corda graduada, e a velocidade da água com um fluxômetro General Oceanics 2030.

\section{Análises dos dados}

Primeiramente foi calculada a curva de saturação de espécies (MagurRan 2004) por intermédio do método Jacknife e comparada à riqueza observada utilizando-se o programa Biodiversity Professional ${ }^{\odot}$. Posteriormente, foi realizada uma comparação entre as assembléias de peixes amostradas nos riachos e na calha principal do ribeirão Ouvidor considerando-se a: a) riqueza, ou seja, o número de espécies; b) abundância, isto é, o número total de indivíduos capturados; c) Diversidade de Shannon-Wiener (Magurran 2004), utilizando-se a fórmula: $\mathrm{H}^{\prime}=-\Sigma$ (pi) $\left(\log _{2} \mathrm{pi}\right)$, onde: $\mathrm{H}^{\prime}$ = índice de diversidade de espécies de Shannon-Wiener, pi = proporção de indivíduos encontrados em uma dada espécie e $\log _{2} \mathrm{pi}=\log$ aritmo na base 2 de pi; e uniformidade, utilizando o índice de Pielou (Magurran 2004): J' = H'/ $\log _{2}$ S, onde: J' = índice de uniformidade de Pielou, $\mathrm{H}^{\prime}$ = índice de diversidade de Shannon-Wiener e $\log _{2} \mathrm{~S}=\log$ aritmo na base 2 da riqueza.

Para o cálculo da diversidade e da uniformidade da bacia foi considerado o conjunto de amostras, enquanto que para cada trecho amostral foi considerada a média desses descritores. Para determinar a diferença estatística entre os trechos amostrados, com relação a esses descritores, foi utilizada uma análise de ANOVA seguida de um teste de comparação múltipla (Bonferroni). Os trechos amostrados foram comparados a um valor de referência (diversidade e uniformidade da bacia) por intermédio de um teste de " $\mathrm{t}$ " (One-Sample). Em ambos os casos foram utilizados o programa SYSTAT ${ }^{\circledR}$.

Relação riqueza/abundância (curva de rarefação) (MAgurRan 2004), calculada para determinar as diferenças entre os trechos de amostragem através da estimativa da riqueza utilizando-se o programa Biodiversity Professional @ C. Para tanto foi considerado o ponto de corte equivalente à menor abundância encontrada nos trechos amostrados (120 indivíduos no Córrego Riacho). Esta análise é apropriada quando as amostras incluem um diferente número de indivíduos, como é o caso neste estudo, sendo que subamostras de igual tamanho de indivíduos são retiradas aleatoriamente e assim é obtida uma estimativa de riqueza, o que torna as amostras comparáveis entre si (Ricklefs 1996, MagurRan 2004).

Posteriormente, a riqueza estimada pela análise de rarefação, a largura e a velocidade foram submetidas a uma análise de correlação de Pearson do programa Statistica 6.0.

Constância (DAJOz 1978), utilizada para determinar quais as espécies constantes numa escala espacial (a) e temporal (b): $\mathrm{C}=\mathrm{n} / \mathrm{N} * 100$, onde em $(\mathrm{a}): \mathrm{n}=$ número de riachos onde $\mathrm{a}$ espécie foi coletada e $\mathrm{N}=$ número total de riachos amostrados na bacia; e em (b) $\mathrm{n}$ = número de coletas onde a espécie foi capturada e $\mathrm{N}$ = número total de coletas realizadas nos trechos.

A partir do resultado obtido em ambas as análises, cada espécie foi classificada seguindo DAJOz (1978) em: constante, C $>50 \%$; acessória, $25 \% \leqslant \mathrm{C} \leqslant 50 \%$; e acidental, $\mathrm{C}<25 \%$.

\section{RESULTADOS}

Na bacia do ribeirão Ouvidor foram capturados 4049 indivíduos distribuídos em 35 espécies, seis ordens e 14 famílias (Tab. II). Dentre as seis ordens encontradas, a que apresentou maior riqueza de espécies foi Characiformes (16 espécies), seguida de Siluriformes (13 espécies). Os Cyprinodontiformes, os Gymnotiformes e os Synbranchiformes apresentaram apenas uma espécie. A família mais abundante foi Characidae, com 1987 indivíduos, seguida de Poeciliidae com 725 espécimes, e Curimatidae com apenas um indivíduo. 
Tabela II. Abundância absoluta e relativa das espécies de peixes coletadas na bacia do ribeirão Ouvidor, alto rio Paraná, Goiás entre agosto de 2004 e junho de 2005.

Espécie $\quad$ Abundância absoluta $\quad$ Abundância relativa (\%)

Characiformes

Parodontidae

Apareiodon ibitiensis Amaral Campos, 1944

Apareiodon piracicabae (Eigenman, 1907)

Parodon nasus Kner, 1859

Curimatidae

Cyphocharax modestus (Fernández-Yépez, 1948)

Anostomidae

Leporinus microphthalmus Garavello, 1989

Crenuchidae

Characidium fasciatum Reinhardt, 1866

Characidium gomesi Travassos, 1956

Characidae

Astyanax altiparanae Garutti \& Britski, 2000

Astyanax eigenmanniorum (Cope, 1894)

Astyanax cf. fasciatus Cuvier, 1819

Bryconamericus sp.

Bryconamericus sp. 2

Planaltina myersi Böhlke, 1954

Hyphessobrycon sp.

Piabina argentea Reinhardt, 1867

Erythrinidae

Hoplias malabaricus (Block, 1794)

Siluriformes

Trichomycteridae

Trichomycterus sp.

Loricariidae

Hisonotus sp.

Hypostomus ancistroides (Ihering, 1911)

Hypostomus margaritifer (Regan, 1908)

Hypostomus sp. 1

Hypostomus sp. 2

Hypostomus regani (Ihering, 1905)

Neoplecostomus paranensis Langeani, 1990

Heptapteridae

Cetopsorhamdia cf. iheringi Schubart \& Gomes, 1959

Phenacorhamdia sp.

Rhamdia quelen (Quoy \& Gaimard, 1824)

Imparfinis sp.

Pimelodidae

Pimelodus sp.

$\begin{array}{rr}190 & 4,70 \\ 8 & 0,20 \\ 10 & 0,25 \\ 172 & 4,25 \\ 1 & 0,02 \\ 1 & 0,02 \\ 5 & 0,12 \\ 5 & 0,12 \\ 170 & 4,20 \\ 168 & 4,15 \\ 2 & 0,05 \\ 1987 & 49,07 \\ 650 & 16,05 \\ 76 & 1,88 \\ 762 & 18,82 \\ 111 & 2,74 \\ 17 & 0,42 \\ 9 & 0,22 \\ 14 & 0,35 \\ 348 & 8,59 \\ 46 & 1,14 \\ 46 & 1,14\end{array}$

60

1,48

1,48

3,10

0,25

0,15

1,46

0,47

0,37

0,15

0,25

3,75

1,11

0,02

3,68

0,05

0,30

0,30 
Tabela II. Continuação.

\begin{tabular}{lcc}
\hline \multicolumn{1}{c}{ Espécie } & Abundância absoluta & Abundância relativa (\%) \\
\hline Gymonotiformes & & 37 \\
Gymnotidae & 147 & 3,63 \\
Gymnotus aff. carapo Linnaeus, 1758 & & 3,63 \\
Cyprinodontiformes & 725 & 17,91 \\
Poeciliidae & 725 & 17,91 \\
$\quad$ Poecilia reticulata Peters, 1859 & & 0,10 \\
Synbranchiformes & 4 & 0,10 \\
Synmbrachidae & 4 & 9,39 \\
Synbranchus marmoratus Bloch, 1795 & & 8,40 \\
Perciformes & 380 & 0,22 \\
Cichlidae & 340 & 0,77 \\
Cichlasoma paranaense Kullander, 1983 & 9 & 31 \\
Tilapia rendalli (Boulenger, 1897) & & \\
\hline
\end{tabular}

A análise da constância espacial indica que duas espécies estiveram presentes em todos os riachos: Astyanax cf. fasciatus Cuvier, 1819 e Rhamdia quelen Quoy \& Gaimard, 1824, e 11 em pelo menos seis riachos, totalizando $37,1 \%$ de espécies constantes; 12 espécies (34,3\%) são acessórias e 10 (28,6\%), coletadas no máximo em dois riachos, são acidentais (Tab. III).

As espécies mais abundantes na bacia do ribeirão Ouvidor foram Astyanax cf. fasciatus (762 indivíduos), Poecilia reticulata Peters, 1859 (725) e A. altiparanae Garutti \& Britski, 2000 (650) (Tab. II).

A comparação entre o número máximo de espécies estimadas (Jacknife) e observadas mostrou que ambas foram similares (39,5 e 35, respectivamente) (Fig. 2).

A diversidade de espécies de peixes variou espacialmente entre os trechos amostrados $(\mathrm{p}=0,001)$. Isto devido principalmente às diferenças da diversidade entre o ribeirão Ouvidor e os riachos Posse dos Rodrigues $(\mathrm{p}=0,004)$ e Taquara I ( $\mathrm{p}=$ 0,000); Taquara I e os cursos de água Sapê $(p=0,019)$, Santo Antônio ( $p=0,023)$ e Taquara II $(p=0,005)$ (Tab. IV). A uniformidade não apresentou diferenças significativas $(p=0,137)$.

A diversidade de espécies de cada trecho amostrado em relação à da bacia do ribeirão Ouvidor apresentou diferenças significativas em todos os casos $(p<0,05)$ (Tab. V). A uniformidade foi diferente entre a bacia e os trechos do canal principal ( $p=0,028)$ e do riacho Taquara II $(p=0,005)$ (Tab. V).

A análise de rarefação indica que a riqueza foi maior nos trechos localizados nos riachos Ouvidor (19 espécies), Lagoa (17), Posse dos Rodrigues (15), Riacho (14), Taquara II (12), Santo Antônio (11), Olhos d'água (11) e Sapê (11), que nos amostrados no Buraco (9) e Taquara I (7) (Fig. 3). Estes resultados estão correlacionados com a largura da calha $(\mathrm{r}=0,77$; Tab. VI), ou seja, trechos com a calha larga $(>2,4$ e $<9 \mathrm{~m})$ apresentam maior riqueza $(\geqslant 11 \mathrm{e} \leqslant 19)$, enquanto que nos trechos estreitos $(<2,2 \mathrm{~m})$ o número de espécies é menor $(\leqslant 9)$.

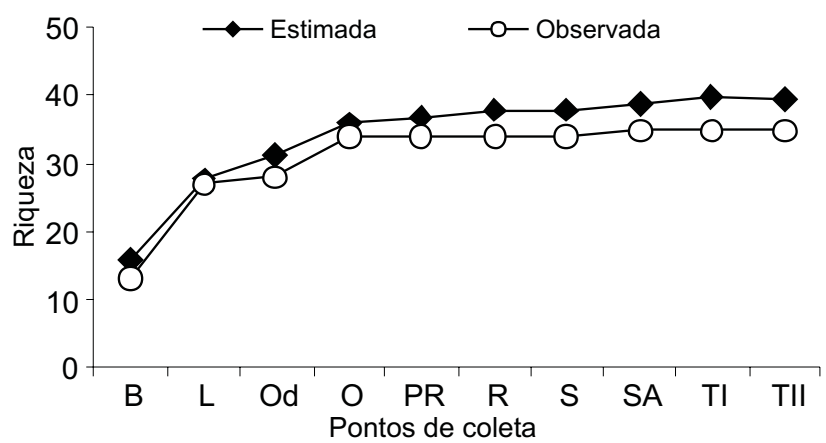

Figura 2. Comparação entre a riqueza observada e estimada resultante da análise de Jacknife. (B) Buraco, (L) Lagoa, (Od) Olhos d'água, (O) Ouvidor, (PR) Posse dos Rodrigues, (R) Riacho, (S) Sapê, (AS) Santo Antônio, (TI) Taquara I, (TII) Taquara II.

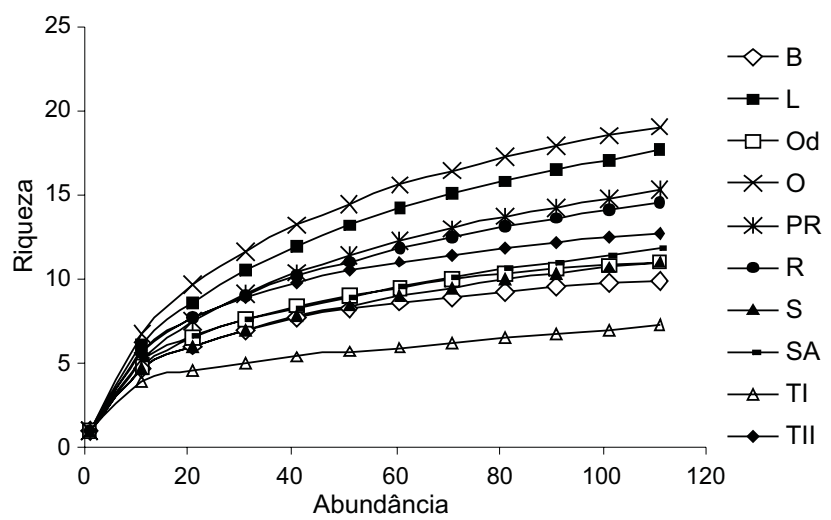

Figura 3. Curva de rarefação por trecho amostral. (B) Buraco, (L) Lagoa, (Od) Olhos d'água, (O) Ouvidor, (PR) Posse dos Rodrigues, (R) Riacho, (S) Sapê, (AS) Santo Antônio, (TI) Taquara I, (TII) Taquara II. 
Tabela III. Classificação quanto à constância das espécies capturadas nos trechos dos riachos da bacia do ribeirão Ouvidor. (B) Buraco, (L) Lagoa, (Od) Olhos d'água, (O) Ouvidor, (PR) Posse dos Rodrigues, (R) Riacho, (S) Sapê, (AS) Santo Antônio, (TI) Taquara I, (TII) Taquara II, (AC) acidental, (AE) acessória, (CO) constante.

\begin{tabular}{|c|c|c|c|c|c|c|c|c|c|c|}
\hline \multirow{2}{*}{ Espécies } & \multicolumn{10}{|c|}{ Cursos de água } \\
\hline & B & $\mathrm{L}$ & Od & $\mathrm{O}$ & $\mathrm{PR}$ & $\mathrm{R}$ & $S$ & SA & $\mathrm{TI}$ & TII \\
\hline Apareiodon ibitiensis & - & - & - & $A C$ & $A C$ & - & - & $A C$ & - & - \\
\hline Apareiodon piracicabae & - & - & - & $\mathrm{AC}$ & $A C$ & - & $\mathrm{AC}$ & $\mathrm{CO}$ & - & - \\
\hline Astyanax altiparanae & - & $A C$ & - & $\mathrm{CO}$ & $\mathrm{CO}$ & $\mathrm{CO}$ & $\mathrm{CO}$ & $\mathrm{CO}$ & $\mathrm{CO}$ & $\mathrm{CO}$ \\
\hline Astyanax eigenmanniorum & $A C$ & $A C$ & $A C$ & $A C$ & $A C$ & $A C$ & - & - & - & $A C$ \\
\hline Astyanax cf. fasciatus & $\mathrm{CO}$ & $\mathrm{CO}$ & $\mathrm{CO}$ & $\mathrm{CO}$ & $\mathrm{CO}$ & $\mathrm{CO}$ & $\mathrm{CO}$ & $\mathrm{CO}$ & AC & $\mathrm{CO}$ \\
\hline Bryconamericus sp. & - & $A C$ & - & $\mathrm{CO}$ & $\mathrm{CO}$ & $\mathrm{AE}$ & $\mathrm{AE}$ & $\mathrm{CO}$ & AC & $\mathrm{CO}$ \\
\hline Bryconamericus sp. 2 & - & - & - & $A C$ & - & - & $A C$ & $A C$ & AC & AC \\
\hline Cetopsorhamdia cf. iheringi & - & $\mathrm{CO}$ & $\mathrm{CO}$ & $A C$ & - & $\mathrm{AE}$ & - & $A C$ & - & - \\
\hline Characidium fasciatum & $\mathrm{CO}$ & - & - & AC & - & AC & - & - & $A C$ & - \\
\hline Characidium gomesi & AC & - & - & - & - & - & - & - & - & - \\
\hline Cichlasoma paranaense & $\mathrm{CO}$ & - & - & $\mathrm{CO}$ & $\mathrm{CO}$ & $\mathrm{CO}$ & $\mathrm{CO}$ & $\mathrm{CO}$ & $\mathrm{CO}$ & $\mathrm{CO}$ \\
\hline Cyphocarax modestus & - & - & - & - & - & - & - & $A C$ & - & - \\
\hline Gymnotus aff. carapo & - & $\mathrm{CO}$ & - & $\mathrm{AE}$ & $\mathrm{CO}$ & $A C$ & $\mathrm{CO}$ & $A C$ & $\mathrm{CO}$ & $\mathrm{CO}$ \\
\hline Hisonotus sp. & - & $A C$ & $A C$ & $A C$ & - & $A C$ & - & - & - & - \\
\hline Hoplias malabaricus & $\mathrm{CO}$ & - & - & - & $\mathrm{CO}$ & - & $\mathrm{CO}$ & $A C$ & $\mathrm{CO}$ & $\mathrm{AE}$ \\
\hline Hypostomus ancistroides & - & $A C$ & - & AC & $\mathrm{AC}$ & - & - & - & - & - \\
\hline Hyphessobrycon sp. & $A C$ & - & $A C$ & - & - & - & - & AC & - & - \\
\hline Hypostomus margaritifer & - & $\mathrm{AE}$ & $\mathrm{CO}$ & $\mathrm{CO}$ & $\mathrm{AE}$ & $A C$ & $\mathrm{AE}$ & - & - & - \\
\hline Hypostomus sp. 1 & - & $A C$ & - & $\mathrm{AE}$ & AC & $A C$ & - & - & - & - \\
\hline Hypostomus sp. 2 & - & $\mathrm{AE}$ & $A C$ & $\mathrm{AE}$ & AC & - & $A C$ & $A C$ & - & - \\
\hline Hypostomus regani & - & - & - & AC & - & - & $A C$ & - & - & - \\
\hline Imparfinis sp. & - & $A C$ & - & - & - & - & - & - & - & - \\
\hline Laetacara sp. 1 & $\mathrm{AE}$ & - & - & - & - & - & - & - & - & - \\
\hline Leporinus microphtalmus & - & - & - & $A C$ & - & - & - & - & - & $\mathrm{AE}$ \\
\hline Neoplecostomus paranensis & - & - & $\mathrm{AE}$ & - & AC & - & - & - & - & - \\
\hline Parodon nasus & - & $\mathrm{CO}$ & $\mathrm{CO}$ & $\mathrm{AE}$ & AC & $A C$ & $\mathrm{CO}$ & $\mathrm{CO}$ & - & $\mathrm{CO}$ \\
\hline Phenacorhamdia sp. & - & $A C$ & - & - & - & - & - & - & - & - \\
\hline Piabina argentea & - & $\mathrm{CO}$ & $\mathrm{CO}$ & $\mathrm{CO}$ & $\mathrm{CO}$ & $\mathrm{CO}$ & $\mathrm{CO}$ & $\mathrm{CO}$ & - & $\mathrm{CO}$ \\
\hline Pimelodus sp. & $A C$ & $\mathrm{CO}$ & $A C$ & - & AC & - & - & - & - & - \\
\hline Planaltina myersi & - & - & - & $A C$ & - & $A C$ & - & $\mathrm{AE}$ & - & $\mathrm{AE}$ \\
\hline Poecilia reticulata & $\mathrm{CO}$ & $A C$ & - & $\mathrm{CO}$ & $\mathrm{CO}$ & - & $\mathrm{CO}$ & $\mathrm{CO}$ & $\mathrm{AE}$ & $\mathrm{CO}$ \\
\hline Rhamdia quelen & $\mathrm{CO}$ & $\mathrm{AE}$ & $\mathrm{CO}$ & $\mathrm{CO}$ & $\mathrm{CO}$ & $\mathrm{CO}$ & $\mathrm{CO}$ & $\mathrm{CO}$ & $\mathrm{AE}$ & $\mathrm{AE}$ \\
\hline Synbranchus marmoratus & - & $A C$ & - & - & AC & - & - & $\mathrm{AE}$ & - & - \\
\hline Tilapia rendalli & $\mathrm{AE}$ & - & - & - & - & - & - & $\mathrm{CO}$ & - & - \\
\hline Trichomycterus sp. & $\mathrm{CO}$ & - & $A C$ & - & - & - & - & - & - & - \\
\hline
\end{tabular}


Tabela IV. Análise de variância com relação à diversidade entre os trechos dos cursos de água amostrados. Valores significativos estão em negrito ( $p<0,05)$. (B) Buraco, (L) Lagoa, (Od) Olhos d'água, (O) Ouvidor, (PR) Posse dos Rodrigues, (R) Riacho, (S) Sapê, (AS) Santo Antônio, (TI) Taquara I, (TII) Taquara II.

\begin{tabular}{|c|c|c|c|c|c|c|c|c|c|c|}
\hline & B & $\mathrm{L}$ & Od & $\mathrm{O}$ & $R$ & PR & $S$ & SA & $\mathrm{TI}$ & TII \\
\hline$B$ & 1,000 & & & & & & & & & \\
\hline $\mathrm{L}$ & 1,000 & 1,000 & & & & & & & & \\
\hline Od & 1,000 & 1,000 & 1,000 & & & & & & & \\
\hline $\mathrm{O}$ & 0,067 & 1,000 & 0,060 & 1,000 & & & & & & \\
\hline $\mathrm{R}$ & 1,000 & 1,000 & 1,000 & 0,250 & 1,000 & & & & & \\
\hline PR & 1,000 & 1,000 & 1,000 & 0,004 & 1,000 & 1,000 & & & & \\
\hline$S$ & 1,000 & 1,000 & 1,000 & 1,000 & 1,000 & 0,694 & 1,000 & & & \\
\hline SA & 1,000 & 1,000 & 1,000 & 1,000 & 1,000 & 0,802 & 1,000 & 1,000 & & \\
\hline TI & 0,965 & 0,057 & 1,000 & 0,000 & 0,293 & 1,000 & 0,019 & 0,023 & 1,000 & \\
\hline TII & 1,000 & 1,000 & 1,000 & 1,000 & 1,000 & 0,237 & 1,000 & 1,000 & 0,005 & 1,000 \\
\hline
\end{tabular}

A análise da constância temporal indica que o trecho do riacho Santo Antônio apresentou a maior quantidade de espécies constantes (10). As espécies classificadas como acessórias foram encontradas, em maior quantidade, no Ouvidor e Taquara II (quatro em ambos os trechos), enquanto que pontos amostrados nos riachos Ouvidor, Posse dos Rodrigues e Lagoa apresentaram os maiores números de espécies acidentais (11, 10 e 10 , respectivamente) (Tab. III).

\section{DISCUSSÃO}

A ictiofauna neotropical apresenta uma alta diversidade e riqueza de espécies, com um número estimado entre 5000 (Agostinho et al. 2005) e 6025 espécies (Reis et al. 2003). No entanto, aproximadamente 30 a $40 \%$ da ictiofauna de águas interiores ainda não foi descrita (AgostinHo et al. 2005). Este pode ser o caso de algumas espécies neste estudo, onde $40 \%$ das mesmas foram identificadas ao nível do gênero, o que revela a necessidade de estudos taxonômicos da ictiofauna no alto da bacia do rio Paraná em Goiás.

Nesta bacia foi registrada uma riqueza de 35 espécies, incluindo duas espécies exóticas ( $6 \%$ do total): Poecilia reticulata, nativa da Venezuela, do norte do Brasil e das Guianas e Tilapia rendalli originária do continente africano. Este valor é próximo ao da riqueza estimada (39,5 espécies). Esta riqueza é menor quando comparada às 48 espécies coletadas nos afluentes do ribeirão João Leite, bacia do Paranaíba (A.P. FIALHO, comunicação pessoal), apesar do mesmo esforço amostral e uso da mesma técnica de captura durante um período sazonal similar (estiagem e chuva). Entretanto, é necessário notar que no referido estudo o número de cursos de água amostrados foi maior (22) (A.P. Fialho, comunicação pessoal) que os deste estudo (10), havendo assim influência da área amostrada sobre o número de espécies coletadas como é indicado pelo estudo de BragA \& ANDRADE (2005) na microbacia do ribeirão Grande. Além disso, é importante destacar que a bacia do João Leite possui uma área maior que a do Ouvidor, ou seja, apresenta uma maior heterogeneidade ecológica proporcionando oportunidades de amostrar mais tipos de habitats e conseqüentemente mais espécies (Ricklefs 1996), além de abranger uma área de preservação, o Parque Estadual Altamiro de Moura Pacheco, o que não ocorre na bacia em estudo, onde a paisagem impactada é predominante devido, principalmente, à presença de áreas de pastagem e indústria.

Na ictiofauna da bacia do ribeirão Ouvidor houve predominância das ordens Characiformes e Siluriformes. As mesmas são dominantes nos sistemas fluviais sul-americanos (VARI \& Malabarba 1998, Castro 1999, Lowe-McConnell 1999). Esta predominância também foi observada por FIALHO et al. (2007) nos riachos do rio Meia Ponte da bacia do Paranaíba, e por BenediTO-Céílio et al. (2004) em três tributários do Paranaíba.

Lemes \& GarutTi (2002) afirmam que a constância reflete a habilidade biológica que a espécie tem em explorar os recursos ambientais disponíveis num determinado momento no biótopo. Uma destas habilidades estaria relacionada com o hábito alimentar onívoro, o qual permite a exploração de alimentos tanto de origem animal como vegetal (CASSEMIro et al. 2002). Este seria o caso, neste estudo, de Astyanax cf. fasciatus que pertence a um gênero no qual a maioria das espécies é onívora - Astyanax altiparanae (CASSEMIRo et al. 2002, Gomiero \& Braga 2003, Dias et al. 2005, Bennemann et al. 2005), Astyanax eigenmanniorum Cope, 1894 (Bennemann et al. 2005), Astyanax cf. fasciatus (BENNEMANN et al. 2005), Astyanax scabripinnis Jenyns, 1842 (BENNEMANn et al. 2005) e de Rhamdia quelen, um bagre de hábitos onívoros (Fracalossi et al. 2004). O hábito alimentar insetívoro de Gymnotus aff. carapo e onívoro com tendência a insetívoro de Poecilia reticulata (CASTRo \& CASATTI 1997, LemES \& GARUTT 2002) também explicaria a constância destas espécies em alguns dos trechos amostrados, nos quais são encontrados remanescentes da mata ripária que permitem a obtenção de alimento (alóctone) por parte dessas espécies (FIALHo \& TejerINAGARro 2004). Por sua vez, a substituição da mata ripária por pastagens explicaria a baixa quantidade de espécies constantes 
Tabela V. Abundância absoluta, riqueza (S), valores médios e desvio padrão (entre parênteses) da diversidade de Shannon-Wiener ( $\mathrm{H}^{\prime}$ ) e da uniformidade $(U)$ por trecho amostral dos peixes coletados na bacia do ribeirão Ouvidor entre agosto/2004 e junho/2005. (n) Número de amostras, $(p)$ valores do teste de " $\mathrm{t}$ " da comparação dos índices de diversidade e uniformidade de cada trecho amostral com estes da bacia. Os valores significativos $(p<0,05)$ são indicados em negrito.

\begin{tabular}{|c|c|c|c|c|c|c|c|}
\hline \multirow{2}{*}{ Local } & \multirow{2}{*}{ Curso de água } & \multirow{2}{*}{ Abundância } & \multirow{2}{*}{$S$} & \multirow{2}{*}{$\mathrm{H}^{\prime}$} & \multirow{2}{*}{$U$} & \multicolumn{2}{|c|}{$\mathrm{p}$} \\
\hline & & & & & & $\mathrm{H}^{\prime}$ & $\mathrm{U}$ \\
\hline \multirow[t]{3}{*}{ Canal principal } & Ouvidor & 240 & 23 & 2,864 & 0,864 & 0,008 & 0,028 \\
\hline & & & & $(0,351)$ & $(0,104)$ & & \\
\hline & & & & $\mathrm{n}=5$ & & & \\
\hline \multirow[t]{27}{*}{ Afluentes } & Buraco & 1242 & 13 & 2,033 & 0,709 & 0,000 & 0,950 \\
\hline & & & & $(0,384)$ & $(0,092)$ & & \\
\hline & & & & $n=6$ & & & \\
\hline & Lagoa & 143 & 19 & 2,291 & 0,801 & 0,001 & 0,102 \\
\hline & & & & $(0,459)$ & $(0,116)$ & & \\
\hline & & & & $\mathrm{n}=6$ & & & \\
\hline & Olhos d'água & 278 & 13 & 1,986 & 0,733 & 0,001 & 0,615 \\
\hline & & & & $(0,388)$ & $(0,108)$ & & \\
\hline & & & & $\mathrm{n}=5$ & & & \\
\hline & Posse dos Rodrigues & 347 & 20 & 2,148 & 0,693 & 0,000 & 0,807 \\
\hline & & & & $(0,377)$ & $(0,128)$ & & \\
\hline & & & & $n=6$ & & & \\
\hline & Riacho & 120 & 15 & 1,762 & 0,793 & 0,004 & 0,138 \\
\hline & & & & $(0,688)$ & $(0,104)$ & & \\
\hline & & & & $\mathrm{n}=5$ & & & \\
\hline & Sapê & 541 & 15 & 2,367 & 0,760 & 0,001 & 0,242 \\
\hline & & & & $(0,429)$ & $(0,097)$ & & \\
\hline & & & & $\mathrm{n}=6$ & & & \\
\hline & Santo Antônio & 649 & 20 & 2,382 & 0,717 & 0,000 & 0,703 \\
\hline & & & & $(0,253)$ & $(0,061)$ & & \\
\hline & & & & $\mathrm{n}=6$ & & & \\
\hline & Taquara I & 269 & 10 & 1,206 & 0,627 & 0,000 & 0,635 \\
\hline & & & & $(0,683)$ & $(0,354)$ & & \\
\hline & & & & $n=6$ & & & \\
\hline & Taquara II & 220 & 14 & 2,483 & 0,859 & 0,000 & 0,005 \\
\hline & & & & $(0,238)$ & $(0,079)$ & & \\
\hline & & & & $\mathrm{n}=6$ & & & \\
\hline Bacia & & 4049 & 35 & 3,627 & 0,707 & - & - \\
\hline
\end{tabular}

numa escala temporal no riacho Taquara I, tendo em vista que zonas ripárias intensamente alteradas empobrecem a cobertura vegetal e os habitats disponíveis e diminuem a diversidade e complexidade da biota aquática (PINTo et al. 2006), incluindo a ictiofauna.

Por outro lado, é necessário considerar a influência da distribuição geográfica de cada espécie, como por exemplo, Rhamdia quelen, a qual tem sua ocorrência registrada desde a região central da Argentina até o sul do México (Fracalossi $e t$ al. 2004) e Astyanax altiparanae, a qual se distribui amplamente na bacia do Paraná (Gomiero \& Braga 2003, Fialho \& TejerinaGARRo 2004), ou ainda da adaptação que permite explorar ambientes que recebem efluentes domésticos, como é o caso de Astyanax cf. fasciatus (Schulz \& Martins-Júnior 2001) no riacho Santo Antônio. Neste último, a interação (peixes-efluentes) seria responsável pelo maior número de espécies constantes observado, numa escala temporal, considerando que os efluentes domésticos influenciam na disponibilidade de alimento e conseqüentemente sobre as redes tróficas aquáticas incluindo os peixes (DEBRUYn et al. 2003). 
A análise de rarefação indica que a calha principal do ribeirão Ouvidor apresenta uma riqueza elevada de peixes (19 espécies) e baixa abundância (240 indivíduos) associadas a uma alta diversidade (média $=2,864$ bits/indivíduos) e uniformidade (média $=0,864)$. Esta combinação de características ecológicas da comunidade de peixes do ribeirão Ouvidor é diferente das encontradas nos outros riachos. Este resultado pode ser explicado, em parte, pelo comprimento e pela largura da calha do referido ribeirão, fatores estes que influenciam na riqueza (GrenOUILLET et al. 2004) e, por outra, pela maior disponibilidade de habitats no canal principal do mesmo (BRAGA 2004). Entretanto, a relação largura da calha-riqueza também explica os resultados observados nos outros cursos de água amostrados, ou seja, maior riqueza em riachos largos (Riacho, Santo Antônio, Sapê, Lagoa, Posse dos Rodrigues, Taquara II e Olhos d'Água) que em estreitos (Taquara I e Buraco). Porém, a variação da riqueza entre trechos pode também estar relacionada com os impactos causados pelas atividades antropogênicas, às quais estão submetidas as assembléias de peixes localizadas em alguns afluentes (esgotos domésticos no riacho Santo Antônio, mineração no Taquara II, atividades agropecuárias no Olhos d'água).

Impactos de origem antrópica são também observados nos riachos Buraco (fragmentação do habitat aquático) e Taquara I (mata ciliar substituída por pastagens e reservatório a montante do trecho amostrado) e explicam a baixa riqueza encontrada nestes. Entretanto, a riqueza pode estar relacionada com as características biológicas das espécies presentes, como é o caso de Poecilia reticulata. Esta é resistente a condições variadas da qualidade de água (ANDRADE et al. 2005), sendo que em cursos de água que drenam áreas urbanas a sua densidade aumenta em situações de alta concentração de fósforo, menor concentração de oxigênio e baixa profundidade (Cunico et al. 2006), esta última situação observada neste estudo no riacho Buraco.

\section{AGRADECIMENTOS}

Os autores agradecem a diversos alunos de graduação do curso de Biologia/UCG, assim como à equipe do Centro de Biologia Aquática, especialmente Waldeir Francisco de Menezes e Tatiana Lima de Melo pela ajuda nas coletas, à Fundação Aroeira e à Neotropica Tecnologia Ambienttal Ltda. pelo apoio financeiro e logístico, à CAPES pela concessão da bolsa de Mestrado para a primeira autora, e aos avaliadores anônimos pelas sugestões.

\section{REFERÊNCIAS BIBLIOGRÁFICAS}

Agostinho, A.A.; S.M. Thomaz \& L.C. Gomes. 2005. Conservação da biodiversidade em águas continentais do Brasil. Megadiversidade 1 (1): 70-78.

Andrade, R.L.B.; L.S. Andrade; W.R. Boscolo \& C.M. Soares. 2005. Comportamento, sobrevivência e desenvolvimento de lebistes, Poecilia reticulata, submetidos a agentes utilizados na profilaxia de doenças. Acta Scientiarum, Animal Sciences, 27 (4): 523-528.
Benedito-Cecílio, E.; C.V. Minte-Vera; C.H. Zawadzki; C.S. Pavanelli; F.H.G. Rodrigues \& M.F. Gimenes. 2004. Ichthyofauna from the Emas National Park region: composition and structure. Brazilian Journal of Biology 64 (3A): 371-382.

Bennemann, S.T.; A.M. Gealh; M.L. Lorsi \& L.M. Souza. 2005. Ocorrência e ecologia trófica de quatro espécies de Astyanax (Characidae) em diferentes rios da bacia do rio Tibagi, Paraná, Brasil. Iheringia, Série Zoologia, 95 (3): 247-254.

Braga, F.M.S. DE. 2004. Habitat, distribuição e aspectos adaptativos de peixes da microbacia do ribeirão Grande, Estado de São Paulo, Brasil. Acta Scientiarum, Biological Sciences, 26 (1): 31-36.

Braga, F.M.S. de. \& P. De M. Andrade. 2005. Distribuição de peixes na microbacia do ribeirão Grande, Serra da Mantiqueira Oriental, São Paulo, Brasil. Iheringia, Série Zoologia, 95 (2): 121-126.

Cassemiro, F.A.S.; N.S. Hahn \& R. Fugi. 2002. Avaliação da dieta de Astyanax altiparanae Garutti \& Britski, 2000b (Osteichthyes, Tetragonopterinae) antes e após a formação do reservatório de Salto Caxias, Estado do Paraná, Brasil. Acta Scientiarum, Biological Sciences, 24: 419-425.

Castro, R.M.C. 1999. Evolução da ictiofauna de riachos sulamericanos: padrões gerais e possíveis processos causais, $\mathrm{p}$. 139-155. In: E.P. Caramaschi; R. Mazzoni \& P.R. Peres-Neto (Eds). Ecologia de peixes de riacho. Rio de Janeiro, Programa de Pós-Graduação em Ecologia, UFRJ, Série Oecologia Brasiliensis, vol. 5, 260p.

Castro, R.M. \& L. Casatti. 1997. The fish fauna from a small forest stream of the upper Paraná River basin, southeastern Brazil. Ichthyological Explorations Freshwaters 7 (4): 337-352.

Cunico, A.M; A.A. Agostinho \& J.D. Latini. 2006. Influência da urbanização sobre as assembléias de peixes em três córregos de Maringá, Paraná. Revista Brasileira de Zoologia 23 (4): 1101-1110.

DAJoz, R. 1978. Ecologia Geral. Rio de Janeiro, Editora Vozes, 472p. deBruyn, A.M.H.; D.J. Marcogliese \& J.B. Rasmussen. 2003. The role of sewage in a large river food web. Canadian Journal of Fisheries and Aquatic Sciences 60: 1332-1344.

Dias, R.M.; D. Bailly; R.R. Antonio; H.I. Suzuki \& A.A. Agostinho. 2005. Colonization of the Corumbá Reservoir (Corumbá River, Paraná River Basin, Goiás State, Brazil) by the "lambari" Astyanax altiparanae (Tetragonopterinae, Characidae). Brazilian Archives of Biology and Technology 48: 467-476.

Esteves, K.E. \& J. Lobón-Cerviá. 2001. Fish composition and trophic structure of a clear water Atlantic rainforest stream in Southeastern Brazil. Environmental Biology of Fishes 62: 429-440.

Fialho, A.P. \& F.L. Tejerina-Garro. 2004. Peixes da bacia do rio Meia Ponte, GO. Goiânia, Editora da Universidade Católica de Goiás, Série Didática 12, 105p.

Fialho, A.P.; L.G. Oliveira; F.L. Tejerina-Garro \& L.C. Gomes. 2007. Fish assemblage structure in tributaries of the Meia Ponte River, Goiás, Brazil. Neotropical Ichthyology 5 (1): 53-60. 
Fracalossi, D.M.; G. Meyer; F.M. Santamaria; M. Weingartner \& E. Zaniboni-Filho. 2004. Desempenho do jundiá, Rhamdia quelen, e do dourado, Salminus brasiliensis, em viveiros de terra na região sul do Brasil. Acta Scientiarum, Animal Sciences, 26 (3): 345-352.

GaLinkin, M. 2003. GeoGoiás 2002. Brasília, Agência Ambiental do Estado de Goiás, Fundação CEBRAC, PNUMA, SEMARH, $272 \mathrm{p}$.

Gomiero, L.M. \& F.M.S. Braga. 2003. O lambari Astyanax altiparanae (Characidae) pode ser um dispersor de sementes? Acta Scientiarum, Biological Sciences, 25: 353-360.

Grenouillet, G; D. Pont \& C. Hérissé. 2004. Within-basin fish assemblage structure: the relative influence of habitat versus stream spatial position on local species richness. Canadian Journal of Fisheries and Aquatic Sciences 61: 93-02.

Lemes, E.M. \& V. GarutTi. 2002. Ecologia da ictiofauna de um córrego de cabeceira da bacia do alto rio Paraná, Brasil. Iheringia, Série Zoologia, 92 (3): 69-78.

Lowe-McConnell, R.H. 1999. Estudos ecológicos de comunidades de peixes tropicais. São Paulo, EDUSP, 534p.

Luiz, E.A.; A.A. Agostinho; L.C. Gomes \& N.S. Hanh. 1998. Ecologia trófica de peixes em dois riachos da bacia do rio Paraná. Revista Brasileira de Biologia 58 (2): 273-285.

MagurRan, A.E. 2004. Measuring biological diversity. Oxford, Blackwell Science, 256p.

Mazzoni, R.; N. Fenerich-Verani \& E.P. Caramaschi. 2000. A pesca elétrica como técnica de amostragem de populações e comunidades de peixes em rios costeiros do sudeste do Brasil. Revista Brasileira Biologia 60 (2): 205-216.

Novaes-PInTo, M. 1993. Caracterização geomorfológica do Dis- trito Federal, p. 285-320. In: M. Novaes-Pinto (Ed.). Cerrado: Caracterização, ocupação e perspectivas. Brasília, Editora UnB, $2^{\text {a }}$ ed., $657 \mathrm{p}$.

Penczak, T.; A.A. Agostinho \& E.K. Okada. 1994. Fish diversity and community structure in two tributaries of the Paraná river, Paraná State, Brazil. Hydrobiologia 294: 243-251.

Pinto, B.; F. Araujo \& R. Hughes. 2006. Effects of landscape and riparian condition on a Fish Index of Biotic Integrity in a large Southeastern Brazil River. Hydrobiologia 556: 69-83.

Reis, R.E.; O. Kullander \& C.J. Ferraris-Jr. 2003. Check list of the freshwater fishes of South and Central America. Porto Alegre, EDIPUCRS, 742p.

Ribeiro, J.F. 1998. Cerrado: matas de galeria. Planaltina, EMBRAPA, CPAC, 164p.

Ricklefs, R.E. 1996. A economia da natureza. Rio de Janeiro, Guanabara Koogan, $3^{\text {a }}$ ed., 470p.

Schulz, U.H. \& H. Martins-Junior. 2001. Astyanax fasciatus as bioindicator of water pollution of Rio dos Sinos, RS, Brazil. Brazilian Journal of Biology 61: 615-622.

Severi, W; R.G. Hickson \& T.C.F. Maranhão. 1995. Use of electric fishing for fish fauna survey in Southern Brazil. Revista Brasileira de Biologia 55 (4): 651-660.

Shibatta, O.A. \& C.C. Cheida. 2003. Composição em tamanho dos peixes (Actinopterygii, Teleostei) de ribeirões da bacia do rio Tibagi, Paraná, Brasil. Revista Brasileira de Zoologia 20 (3): 469-473.

Vari, R.P. \& L.R. Malabarba. 1998. Neotropical Ichthyology: an Overview. p. 1-11. In: L.R. MaLabarba; R.E. Reis; R.P. Vari; Z.M.S. LuCena \& C. A.S. Lucena. Phylogeny and Classification of Neotropical Fishes. Porto Alegre, EDIPUCRS, 603p.

Recebido em 13.III.2007; aceito em 16.XI.2007. 